Bassom, Andrew P.; Ilchmann, Achim; Voß, Heinrich :

Oxygen Diffusion in Tissue Preparations with Michaelis-Menten Kinetics

Zuerst erschienen in:

Journal of Theoretical Biology 185 (1997), Nr. 1, S. 119-127

DOI: $\underline{10.1006 / \text { ttbi.1996.0298 }}$ 


\title{
Oxygen Diffusion in Tissue Preparations with Michaelis-Menten Kinetics
}

\author{
Andrew P. Bassom*, Achim Ilchmann* $\ddagger$ and Heinrich Voß† \\ * Department of Mathematics, University of Exeter, North Park Road, Exeter, EX4 4QE, \\ U.K. and $\dagger$ Arbeitsbereich Mathematik, Technische Universität Hamburg-Harburg, Kaserner- \\ str. 12, D-21073 Hamburg, Germany
}

(Received on 12 September 1996, Accepted in revised form on 11 October 1996)

\begin{abstract}
A model is introduced for the oxygen consumption in thin vital tissue preparation. The steady uptake kinetics is modelled by a Michaelis-Menten form and for this case it proved that the resulting boundary value problem admits a unique solution for those parameter ranges typical of related physiological experiments. This solution is compared with Otto Warburg's hyperoxia model and with a hypoxia model. Useful and easily computed approximations are derived for the minimum oxygen supply across the tissue and some numerical solutions of the governing equations are discussed.
\end{abstract}

C. 1997 Academic Press Limited

\section{Introduction}

Pharmacological and physiological experiments are being increasingly performed on thin vital tissue preparations known as slices. In contrast to the corresponding problem for spherical cells, the oxygen diffusion characteristics in slices are poorly understood and remain a largely unresolved topic. The first author to study diffusion for spherical cells appears to have been Rashevsky (1960) who modelled the kinetics for oxygen uptake by piecewise linear functions. Lin (1976) argued that this supposed uptake is too simplistic and suggested that a Michaelis-Menten kinetics form would be a far more appropriate model. Lin's results were recomputed by McElwain (1978) and Hiltmann \& Lory (1983) were able to prove that this Michaelis-Menten model for spherical cells possesses exactly one solution. This unique solution can only be precisely determined by recourse to numerical procedures, but useful lower and upper bounds on this true solution were given by Anderson \& Arthurs (1985) and these were improved further by sharp polynomial approximations derived by Asaithambi \& Garner $(1989,1992)$.

The only existing model for thin tissue preparations

${ }_{\ddagger}$ Author to whom correspondence should be addressed. E-mail: ilchmann $(a$ maths.exeter.ac.uk is the long-standing classic suggestion of Otto Warburg (1923) who was concerned with the analysis of oxygen consumption within cancerous tissues. He ignored the kinetics model of Michaelis \& Menten (1913) in favour of an assumption that the consumption is constant across the width of the tissue slice. This hypothesis was prompted by the frequent experimental conditions in which the slice is bathed in a solution which is richly supplied with oxygen. This represents the situation in which a high diffusion coefficient leads to an abundant pool of available oxygen and, in this special case, it is intuitively reasonable that there is little to be gained by appealing to a more complicated uptake form.

However, this having been said, there remains a plethora of situations for which the constant oxygen consumption model is wildly inappropriate. The minimal breadth for intact tissues of many internal organs like the brain, liver, heart and kidney from the mouse, rat, guinea pig and other mammalians is sufficiently large that oxygen diffusion is markedly higher outside the tissue slice than within it. For instance, most experiments with brain tissues use slices with a minimal thickness typically in the range $250-400 \mu \mathrm{m}$ and hence the oxygen diffusion lengthscale in the organ is roughly five times larger than the oxygen supply achieved by capillary distances of only 
30-60 $\mu \mathrm{m}$ [see Metzger et al. (1980) for further discussion]. In order to control the inner oxygen supply via the bath oxygen concentration (which is easily monitored by microelectrodes) a realistic oxygen consumption model is needed. Moreover, Yamamoto (1972) introduced non-pathological brain tissue slices for neurophysiological experiments. For this type of preparation Bingmann \& Kolde (1982) report measurements which show conclusively that Warburg's constant consumption model is inappropriate under normal experimental conditions as a distinctive variation in oxygen consumption within the slice is observed. Finally, we mention that modern experiments in both biology and medicine use tissue slices to simulate hypoxia; that is a situation in which the tissue exhibits a low oxygen consumption. Once more, it seems desirable to replace the constant model with a more realistic one and the Michaelis-Menten form is the natural first choice for this.

These deficiencies in Warburg's analysis provide the motivation for the work in this paper. Our aim is to consider a Michaelis-Menten uptake in a tissue slice and to provide both theoretical and numerical analyses of the resulting equations. Popel (1979) considered the steady-state diffusion in slices with this uptake and examined how the relevant constants in the Michaelis-Menten form can be determined experimentally using the technique proposed by Kawashiro et al. (1975). Here we are able to prove the existence of and derive some useful bounds on the requisite solution and, although the particular problem is not amenable to solution by elementary analytical means, we demonstrate that our bounds are useful for a variety of realistic parameter values. It is convenient to begin by considering a tissue slice within a Cartesian coordinate system which is aligned so that the slice lies in the $Y-Z$ plane with the $X$-axis normal to the slice (see Fig. 1). For simplicity, we

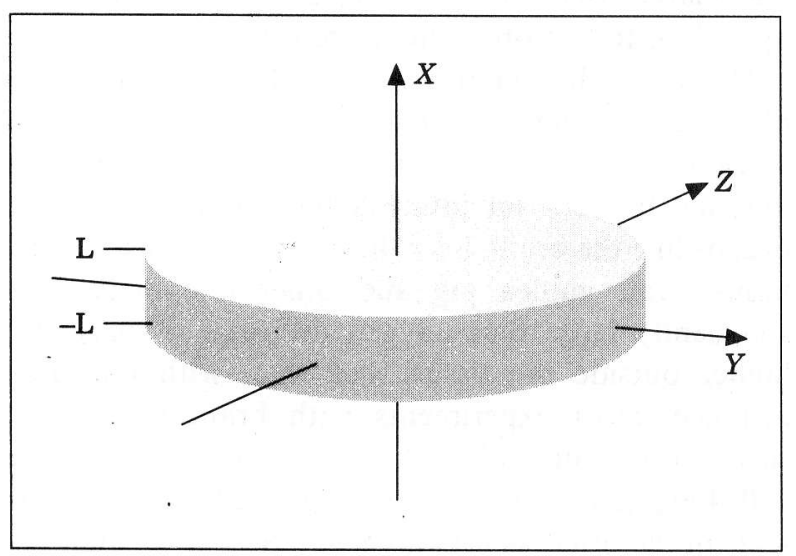

FIG. 1. The geometry of the tissue slice and co-ordinate system. shall assume that the slice is of infinite extent in the $Y$ and $Z$ directions so that edge effects need not be considered. The slice is also taken to lie within $-L \leqslant X \leqslant L$ so that the surrounding oxygen bath lies above the slice where $X>L$ and below it with $X<-L$. If $v(X)$ denotes the oxygen concentration in the tissue then, by Fick's law of diffusion, the uptake equation for the steady state is

$$
D \frac{\mathrm{d}^{2} v}{\mathrm{~d} X^{2}}=q(X),
$$

where $q(X)$ is the oxygen consumption rate and $D$ the constant diffusion coefficient for the tissue. Since oxygen uptake is catalysed by enzymes, this reaction is usually modelled by the Michaelis-Menten kinetics equation

$$
\frac{\mathrm{d}^{2} v}{\mathrm{~d} X^{2}}=\frac{\alpha v}{v+\beta}, \quad-L \leqslant X \leqslant L,
$$

where $\alpha=1 / D$ and $\beta$ denotes the requisite MichaelisMenten constant. This equation needs to be solved subject to the boundary conditions $v(-L)=$ $v(L)=c_{\text {bath }}$ which reflect the fact that at the edges of the tissue the oxygen concentration matches that within the solution surrounding the preparation.

It is useful to scale the problem before proceeding with the analysis. If we define the modified coordinate $x=X / L$ and suppose that $v(X)=c_{\text {bath }} u(x)$ then the model system acquires the form

$$
\frac{\mathrm{d}^{2} u}{\mathrm{~d} x^{2}}=\frac{\gamma u}{u+K} ; \quad u(1)=u(-1)=1,
$$

which needs to be solved for $-1 \leqslant x \leqslant 1$. The solution depends on the parameters

$$
\gamma=\frac{\alpha L^{2}}{c_{\text {bath }}}>0, \quad K=\frac{\beta}{c_{\text {bath }}} \geqslant 0
$$

and we shall sometimes emphasise this dependence by writing the solution to eqn (1) as $u(x ; \gamma, K)$-this is done particularly when we are comparing two solutions relating to different $\gamma$ and $K$ values. At other times we will suppress explicit mention of this parameter dependence when it is less crucial and no confusion should arise.

Two simple limits arise very naturally from eqn (1). In Warburg's hyperoxia model, in which oxygen concentration level within the tissue is high, i.e. $K \ll u(x)$, it is reasonable to approximate $K=0$ in eqn (1). If the corresponding solution of eqn (1) is defined to be $w_{1}(x)$ then

$$
\frac{\mathrm{d}^{2} w_{1}}{\mathrm{~d} x^{2}}=\gamma, \quad w_{1}( \pm 1)=1
$$


and it is immediate that

$$
w_{1}(x)=\frac{1}{2} \gamma\left(x^{2}-1\right)+1 .
$$

We remark that this solution is only physically realistic when $\gamma \leqslant 2$ for otherwise we have $w_{1}<0$ somewhere across the tissue.

On the other hand, if the concentration within the slice is relatively low compared with that in the surrounding bath, then we expect $u \ll K$ and we can then approximate $u(x)$ by $w_{2}(x)$ where

$$
\frac{\mathrm{d}^{2} w_{2}}{\mathrm{~d} x^{2}}=\frac{\gamma w_{2}}{K}, \quad w_{2}( \pm 1)=1
$$

so that

$$
w_{2}(x)=\cosh \left(x \sqrt{\frac{\gamma}{K}}\right) / \cosh \left(\sqrt{\frac{\gamma}{K}}\right) .
$$

In the work below we consider the regions of $\gamma-K$ parameter space in which either (4) or (6) represents a good approximation to $u(x)$. We are also able to obtain simple polynomial approximations for the value of $u$ at the centre of the slice $x=0$ and provide some sample numerical solutions of eqn (1). It is, of course, important to bear in mind the values of $\gamma$ and $K$ which pertain to experimentally realisable conditions. There seems to be a considerable diversity of parameter ranges reported in the literature. According to Bingmann \& Kolde (1982) the rough estimates $\gamma=1$ and $K=0.03$ are realistic for physiological problems. In non-physiological cases both Lin (1976) and McElwain (1978) suggest that $K$ is no more than unity. Under extreme conditions Bingmann \& Kolde (1982) argue that $\gamma$ may be as large as 300 whilst Lin (1976) and McElwain (1978) conclude that with the most unfavourable combination of parameters this upper limit could reach 1600 . However, this latter value is probably unduly pessimistic and, moreover, for our numerical simulations here we shall be principally concentrating on the physiologically more interesting circumstance in which $\gamma=O(1)$.

The remainder of this paper is divided as follows. Section 2 is concerned with a theoretical discussion of eqn (1) and includes mention of existence and uniqueness of solutions and the derivation of lower and upper bounding functions. In writing this article we have been conscious of the diverse backgrounds of the possible readership. For this reason, in Section 2 we have deliberately kept our mathematical techniques as simple as possible whilst consistent with our aim of maintenance of rigour. Those disinterested in the technical details are recommended to only note the main results. In Section 3 we present some numerical solutions of eqn (1) and illustrate how these relate to the theory of the preceding section. In particular, we are able to show how the numerical results of the Michaelis-Menten model compare with the hyperoxia and hypoxia models. Finally, in Section 4 , we make a few concluding remarks.

\section{The Michaelis-Menten, the Warburg (hyperoxia and the Hypoxia Models}

A natural starting point for studying the three models introduced in Section 1 concerns the full Michaelis-Menten problem (1). For ease of presentation, we divide our discussion of this boundaryvalue system into three subsections. In the first two of these we consider various aspects of the problem and in the third we compare the Michaelis-Menten solutions with the simple hyperoxia and hypoxia approximations given by eqns (4) and (6). To begin we must be assured that biologically acceptable solutions of (1) exist and this is the topic we address now.

\subsection{THEORETICAL PROPERTIES OF THE MICHAELIS- MENTEN SOLUTION}

We begin our discussion of the theoretical properties of (1) by introducing a technical lemma which considerably simplifies the ensuing analysis. This lemma, which is concerned with a comparison of functions may be succinctly stated:

\section{Lemma 1}

Let $u(\cdot) \rightarrow[0,1]$ be a solution of eqn (1).

(i) If $w(\cdot)$ satisfies

$$
\frac{\mathrm{d}^{2} w}{\mathrm{~d} x^{2}} \geqslant \frac{\gamma w(x)}{w(x)+K} \text { for all } x \in[-1,1],
$$

together with $w( \pm 1)=1$ then $w(x) \leqslant u(x)$ for all $x \in[-1,1]$.

(ii) If $w(\cdot)$ satisfies

$$
\frac{\mathrm{d}^{2} w}{\mathrm{~d} x^{2}}>\frac{\gamma w(x)}{w(x)+K} \text { for all } x \in[-1,1],
$$

together with $w( \pm 1)=1$ then $w(x)<u(x)$ for all $x \in[-1,1]$.

\section{Proof}

We seek a contradiction by supposing that $w(\xi)>u(\xi)$ for some $\xi \in(-1,1)$. Then by continuity there exists an interval $\left[\xi_{1}, \xi_{2}\right] \subset[-1,1]$ such that $u\left(\xi_{i}\right)=w\left(\xi_{i}\right)$ for $i=1,2$ and $w(x)>u(x)$ for all $x \in\left(\xi_{1}, \xi_{2}\right)$. Hence, we conclude that on the interval $\left[\xi_{1}, \xi_{2}\right]$, due to the monotonicity of the r.h.s.,

$$
\frac{\mathrm{d}^{2} w}{\mathrm{~d} x^{2}} \geqslant \frac{\gamma w(x)}{w(x)+K}>\frac{\gamma u(x)}{u(x)+K}=\frac{\mathrm{d}^{2} u}{\mathrm{~d} x^{2}},
$$

whence $w(\cdot)-u(\cdot)$ is a convex function on $\left[\xi_{1}, \xi_{2}\right]$ 

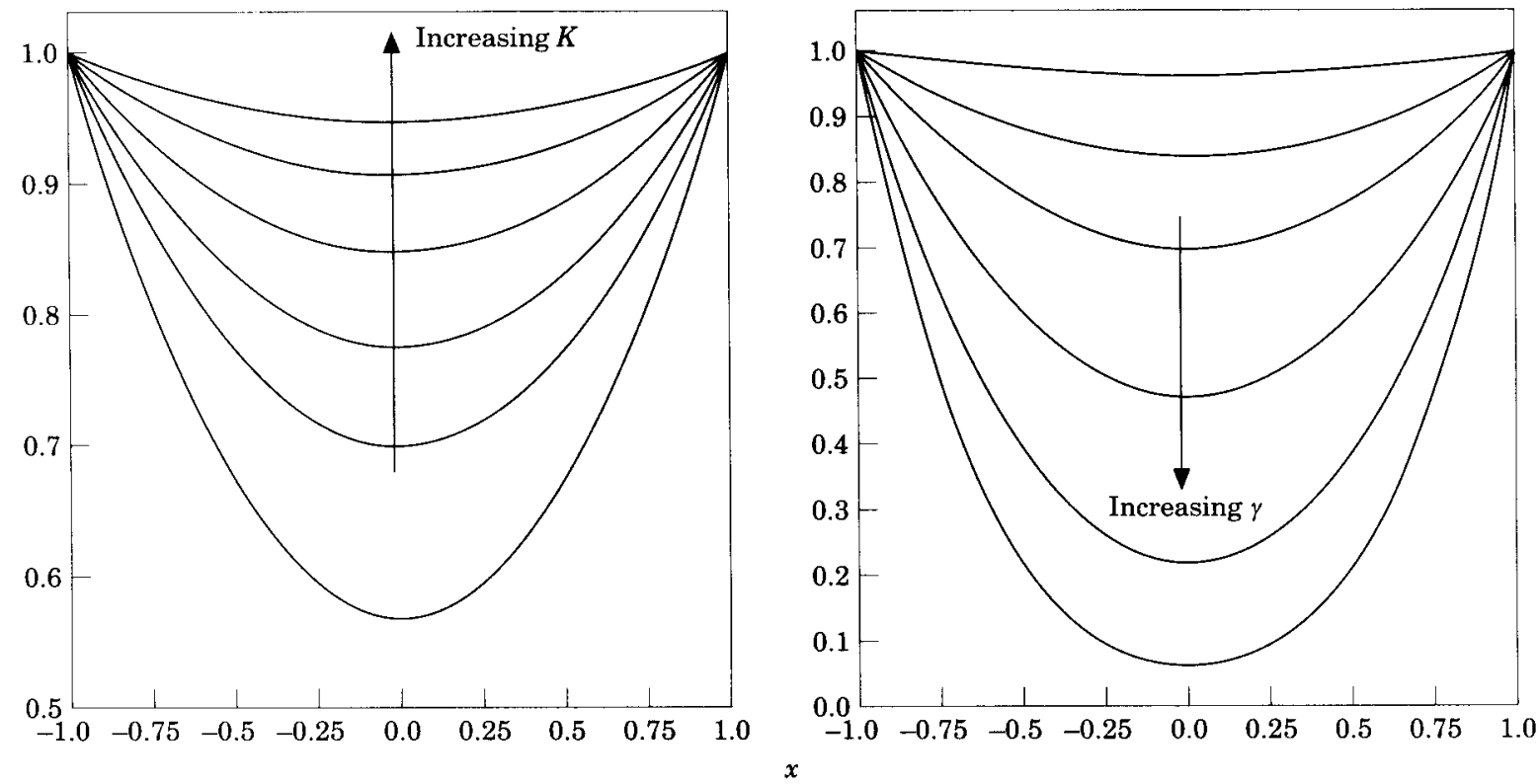

FIG. 2. Numerical solutions $u(\because \gamma, K)$ of the Michaelis-Menten equation (1) for various values of $\gamma$ and $K$. (a) shows the solutions corresponding to $\gamma=1$ and $K=0.1,0.5,1,2,4$ and 8 whilst in (b) are shown solutions for $K=1 / 2, \gamma=0.1,0.5,1,2,4$ and 8 .

with boundary values $w\left(\xi_{i}\right)-u\left(\xi_{i}\right)=0$ for $i=1,2$. Therefore, $w(x)-u(x) \leqslant 0$ for all $x \in\left[\xi_{1}, \xi_{2}\right]$ which contradicts our assumptions so that part (i) of the lemma is proved.

If the inequality is strict, i.e. if

$F(x):=\frac{\mathrm{d}^{2} w}{\mathrm{~d} x^{2}}-\frac{\gamma w(x)}{w(x)+K}>0$ for all $x \in[-1,1]$, then we can choose $\epsilon>0$ sufficiently small so that

$$
\begin{array}{r}
F(x) \geqslant \epsilon\left[2+\frac{\gamma K\left(1-x^{2}\right)}{(\tilde{w}(x)+K)(w(x)+K)}\right] \\
\text { for all } x \in[-1,1]
\end{array}
$$

where

$$
\tilde{w}(x):=w(x)+\epsilon\left(1-x^{2}\right)
$$

An easy calculation shows that for all $x \in[-1,1]$ we have

$$
\frac{\mathrm{d}^{2} \tilde{w}}{\mathrm{~d} x^{2}}=\frac{\gamma w(x)}{w(x)+K}+F(x)-2 \epsilon \geqslant \frac{\gamma \tilde{w}(x)}{\tilde{w}(x)+K},
$$

whence, by (i), $w(x)<\tilde{w}(x) \leqslant u(x)$. This completes the proof.

It is quite straightforward to show that for every $\gamma>0, K>0$ problem (1) admits a solution which is, in fact, unique. Bounds on this solution can be readily evaluated and, for convenience, we can summarise these findings in the following proposition.

\section{Proposition 2}

For every $\gamma, K>0$ there exists a unique solution $u(\cdot) \equiv u(\cdot ; \gamma, K)$ of the boundary value problem (1).
This solution is symmetric about $x=0$, takes on its least value $u_{0}$ at the origin, and satisfies

$$
0<u_{0}<u(x)<1 \text { for all } x \in(-1,1) \backslash\{0\}
$$

and

$$
1-\frac{1}{2} \gamma \leqslant u_{0} \leqslant \bar{\psi}(\gamma, K)<1
$$

where $\psi(\gamma, K)$ is defined by

$$
\begin{aligned}
& \bar{\psi}(\gamma, K)=\frac{1}{2}(1-K)-\frac{1}{4} \gamma \\
& \quad+\left\{\frac{1}{4} K^{2}+\frac{1}{4}(2+\gamma) K+\frac{1}{4}(1-\gamma)+\frac{1}{16} \gamma^{2}\right\}^{12} .
\end{aligned}
$$

Before tackling the proof of these results we show sample solutions of (1) in Fig. 2. Figure 2(a) shows solutions at a fixed value of $\gamma=1$ and various values of $K \in[0.1,8]$ whilst Fig. 2(b) gives results for $K=\frac{1}{2}$ and $\gamma \in[0.1,8]$. These solutions suggest that for fixed $\gamma$ solutions are monotonically increasing with $K$ whilst for prescribed $K$ solutions decrease with $\gamma$ and we shall consider details of this aspect later. The shape of the solutions is as expected: the oxygen concentration in the slice is symmetric about the mass centre and is maximal at the boundaries of the tissue and the bath. The higher the value of $K$, the lower the oxygen consumption within the tissue--an observation which has been reported in many of the experimental results discussed in the introduction. We also see that the value of $u(0)=u_{0}$ is a crucial piece of information as 
the solutions in Fig. 2 demonstrate that the minimum of the oxygen supply to the tissue occurs at $x=0$. Therefore, it is this site at which cells are the first to be insufficiently supplied with oxygen and so die. This will often lead to an uncontrolled non-physiological electrolyte composition of the extracellular space around these cells and so irritate surrounding vital cells.

We also note that it is a simple exercise in differentiation to show that the upper bound $\bar{\psi}$ [see eqn (8b)] on the value $u_{0}$ satisfies $\mathrm{d} \bar{\psi} / \mathrm{d} K>0$ for $K>0$ so that $\bar{\psi}$ is monotonic with $K$. Furthermore, as $K \rightarrow \infty$ so $\bar{\psi} \rightarrow 1$ and hence we conclude that $\bar{\psi}<1$ for all positive values of $\gamma$ and $K$.

\section{Proof of Proposition 2}

We proceed to prove the results of the proposition in two steps.

STEP 1: Existence, uniqueness, symmetry and eqn (7).

For $\alpha \geqslant 0$ consider the initial value problem

$$
\frac{\mathrm{d}^{2} y}{\mathrm{~d} x^{2}}=\frac{\gamma y}{y+K}, \quad y(0)=\alpha, y^{\prime}(0)=0,
$$

which is equivalent to the integral equation

$$
y(x)=(S y)(x):=\alpha+\int_{0}^{x} \int_{0}^{t} \frac{\gamma y(s)}{y(s)+K} \mathrm{~d} s \mathrm{~d} t .
$$

Since $S$ maps the convex and closed set

$$
\left\{y(\cdot) \in \Upsilon[0,1]: 0 \leqslant y(x) \leqslant \alpha+{ }_{2}^{1} \gamma x^{2}, \forall x \in[0,1]\right\}
$$

into itself, we can apply Schauder's Theorem to ensure the existence of a solution to eqn (9). If a solution to $(9)$ is denoted $y(\cdot ; \alpha)$, it is readily seen that $y(\cdot ; 0) \equiv 0$ and $y(x ; 1)>1$ for all $x>0$. Now the continuous dependence on the initial values necessitates the existence of some $\hat{\alpha} \in(0,1)$ such that $y(1 ; \hat{\alpha})=1$ and therefore $y(x ; \hat{\alpha})$ solves the boundary value problem

$$
\frac{\mathrm{d}^{2} y}{\mathrm{~d} x^{2}}=\frac{\gamma y}{y+K}, \quad y^{\prime}(0)=0, y(1)=1 .
$$

By the simple even continuation

$$
s(x):= \begin{cases}y(x ; \hat{\alpha}), & \text { if } x \in[0,1] \\ y(-x ; \hat{\alpha}), & \text { if } x \in-[1,0]\end{cases}
$$

existence of a solution to (1) is guaranteed. Uniqueness is immediate from Lemma 1 and symmetry of the solution follows from the even continuation above.

This information can be used in the process of the derivation of bounds on the solution of (1) which we examine below. At this stage we can also be assured that $u_{0} \neq 0$. If it were not then $u(0)=u^{\prime}(0)=0$ (the latter result from symmetry) and differentiation of (1) shows that all derivatives of $u(x)$ vanish at the origin. This would imply $u(\cdot) \equiv 0$ across the entire tissue slice and this is clearly fallacious.

STEP 2: Derivation of the bounds ( $8 a)$ and $(8 b)$ on the minimal oxygen supply $u_{0}$.

We rewrite eqn (1) as a fixed point problem. With the Green's function for $x, t \in[-1,1]$

$$
g(x, t):= \begin{cases}\frac{1}{2}(x-1)(1+t) & \text { if } t \leqslant x \\ \frac{1}{2}(t-1)(1+x) & \text { if } x \leqslant t\end{cases}
$$

and the operator

$$
(T y)(x):=1+\int_{-1}^{1} \frac{\gamma y(t) g(x, t)}{y(t)+K} \mathrm{~d} t, \quad x \in[-1,1]
$$

it is a straightforward calculation to see that

$$
(T y)^{\prime \prime}(x)=\frac{\gamma y(x)}{y(x)+K}
$$

and hence the boundary value problem (1) is equivalent to $(T u)(x)=u(x)$. Since $g(x, t)$ is nonpositive, the operator $T$ is anti-monotone on the set of positive functions. Therefore, for the solution $u(\cdot)$ of (1),

$$
1+\frac{\gamma u_{0}}{2\left(u_{0}+K\right)}\left(x^{2}-1\right)=\left(T u_{0}\right)(x) \geqslant(T u)(x)=u(x),
$$

setting $x=0$ yields

$$
u_{0}^{2}+\left(K-1+\frac{1}{2} \gamma\right) u_{0}-K \leqslant 0
$$

and completion of the square leads to $u_{0} \leqslant \bar{\psi}$ as defined by ( $8 b)$.

The lower bound on $u_{0}$ as given by ( $8 \mathrm{a}$ ) follows very quickly from Lemma 1 . The hyperoxia model solution $w_{1}(x)$ as given by (3) and (4) satisfies the criteria of that Lemma so that the solution $u(x)$ of eqn (1) satisfies $u(x)>w_{1}(x)$ for all $x \in[-1,1]$. It is obvious that $w_{1}(0)=1-\frac{1}{2} \gamma$ and hence the result $(8 \mathrm{a})$ is immediate.

\subsection{DEPENDENCE OF SOLUTIONS $u(\cdot ; \gamma, K)$ ON $K$ AND $\gamma$}

We have now established upper and lower bounds on the quantity $u_{0}(\gamma, K)$. In order to characterise our solutions further we now turn our attention to study the development of $u(x)$ with varying scaled Michaelis-Menten constant $K$. An important property that we prove below concerns the monotonicity of $u(x)$ : that is, if $u_{1}\left(x ; \gamma, K_{1}\right)$ and $u_{2}\left(x ; \gamma, K_{2}\right)$ are the solutions of eqn (1) for some fixed $\gamma$ but with the choices $K_{2}<K_{1}$ then, for each $x \in(-1,1)$, 
$u_{1}\left(x ; \gamma, K_{1}\right)>u_{2}\left(x ; \gamma, K_{2}\right)$. Graphically, this corresponds to the statement that for each fixed $x$ then $u(x)$ grows with $K$. This phenomenon is evident from Fig. 2(a) for the selection of solutions plotted there and is summarised in the following proposition.

\section{Proposition 3}

For fixed $x \in(-1,1)$, the unique solution $u(x ; \gamma, K)$ of (1) is strictly monotonically decreasing with $\gamma>0$ and increasing with $K>0$.

\section{Proof}

For fixed $\gamma_{1}<\gamma_{2}$ and $K$ we have, for all $x \in[-1,1]$, that

$u^{\prime \prime}\left(x ; \gamma_{2}, K\right)=\gamma_{2} \frac{u\left(x ; \gamma_{2}, K\right)}{u\left(x ; \gamma_{2}, K\right)+K}>\gamma_{1} \frac{u\left(x ; \gamma_{2}, K\right)}{u\left(x ; \gamma_{2}, K\right)+K}$

and therefore Lemma 1(ii) yields

$$
u\left(x ; \gamma_{2}, K\right)>u\left(x ; \gamma_{1}, K\right) .
$$

Strict monotonicity with $K$ is proved in an completely analogous manner and is therefore omitted.

\subsection{COMPARISON BETWEEN $u(x ; \gamma, K)$, THE HYPEROXIA AND THE HYPOXIA MODELS}

In the preceding subsections we have established some fundamental properties of the solution to the Michaelis-Menten equation for the tissue slice. Of significant practical interest is the question of when the solution of the nonlinear system can be reasonably approximated by the explicit hyperoxia or hypoxia solutions $w_{1}(x)$ and $w_{2}(x)$ given by eqns (4)

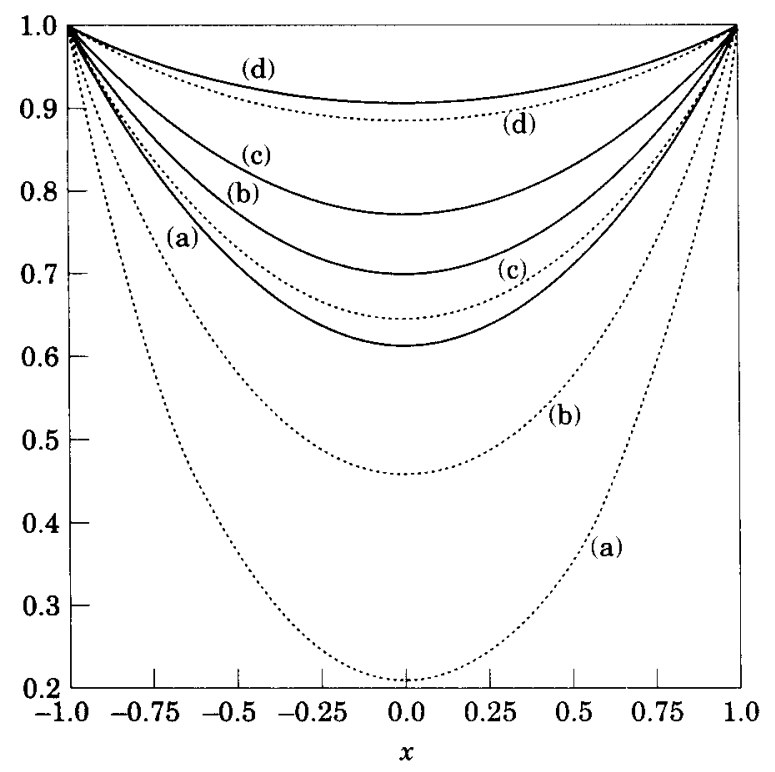

Fig. 3. Comparison of the solutions of the Michaelis-Menten equation (1) and $w_{2}(x)$ as given by eqn (6). Shown are solutions for $\gamma=1$ and (a) $K=0.2$, (b) $K=0.5$, (c) $K=1$ and (d) $K=4$. Solid lines indicate solutions $u(x ; \gamma, K)$ and broken ones show $w_{2}(x ; \gamma, K)$. and (6). In Fig. 3 we compare $u(x)$ and $w_{2}(x)$ for the parameter values $\gamma=1$ and $K=0.2,0.5,1$ and 4 . The results suggest that $w_{2}(x)$ is a useful approximation when $K$ is reasonably large (as expected) but less good for small $K$. It is also obvious that for each parameter combination the actual solution $u(x)$ lies above $w_{2}(x)$ across the whole slice (except, of course, at the edges where the two solutions are the same). Formally, we can summarise this result by

\section{Proposition 4}

For every $\gamma>0, K>0$ and $x \in(-1,1)$ the solutions $u(x ; \gamma, K)$ of $(1), w_{1}(x ; \gamma, K)$ of $(3)$ and $w_{2}(x ; \gamma, K)$ of (6) satisfy

$$
\begin{gathered}
w_{1}(x ; \gamma, K)<u(x ; \gamma, K) \text { if } \gamma \leqslant 2, \\
0<w_{2}(x ; \gamma, K)<u(x ; \gamma, K) .
\end{gathered}
$$

\section{Proof}

Both inequalities (10) and (11) are a consequence of Lemma 1(ii). They are derived using the same arguments as were used in the proof of Proposition 3 and so need not be discussed further.

We can now infer some further bounds on the minimum oxygen concentration $u_{0}$ at the centre of the slice. In view of the bounds in (8a) it is immediate that

$$
0 \leqslant u_{0}-w_{1}(0) \leqslant \bar{\psi}(\gamma, K)-\frac{1}{2} \gamma
$$

and

$$
u_{0}-w_{2}(0) \leqslant \bar{\psi}(\gamma, K)-\left\{\cosh \sqrt{\frac{\gamma}{K}}\right\}^{-1} .
$$

Of greater interest, we can show by considering the function $g(x)=u(x)-w_{1}(x)$ that $g^{\prime}(0)<0$ and $g^{\prime \prime}(x)<0$ for all $x \in[0,1]$ so the difference between $u(x)$ and $w_{1}(x)$ is greatest at the centre of the slice. A similar calculation proves that $u(x)-w_{2}(x)$ is also largest at $x=0$ as long as the parameters satisfy $\sqrt{\gamma}<\sqrt{K} \cosh ^{-1}(1+1 / K)$. The usefulness of bounds (12) is strongly dependent on the value of $K$. If $K$ is small then (12a) represents a tight bound between the Michaelis-Menten solution and the simple parabolic form $w_{1}(x)$ whilst the corresponding result $(12 \mathrm{~b})$ is virtually useless as $w_{2}(x)$ is exponentially small across the overwhelming majority of the slice. In contrast, (12b) is much the better bound for larger $K$ and illustrations of this effect will be given in Section 3 .

\section{Numerical Simulations}

For fixed $\gamma$ and $K>0$ we have obtained a number of key results dealing with the solutions of the 


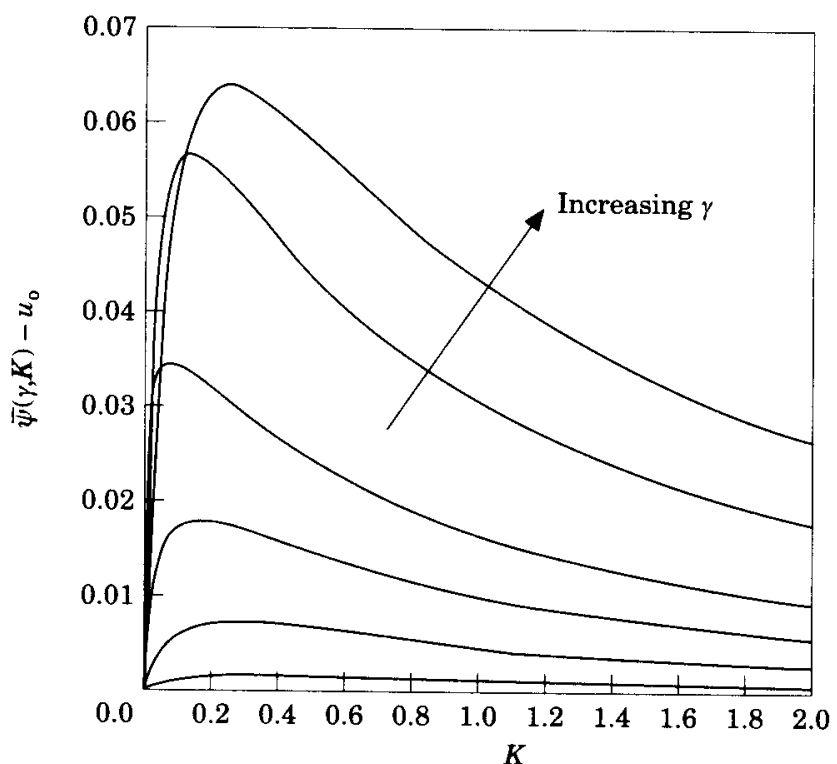

FiG. 4. Graph of the difference between $\psi(\gamma ; K)$ as given by eqn (8b) and the actual oxygen concentration at the centre of the slice $u_{0}=u(0 ; \gamma, K)$. The lines correspond to the parameter values $\gamma=0.5,1,1.5,2,3$ and 4 .

nonlinear boundary-value problem (1). Here we illustrate a number of these by reference to some direct numerical solutions. Our results pertaining to the symmetry and uniqueness of solutions means that for computational purposes our attention can be restricted to the half-slice $0 \leqslant x \leqslant 1$ and once a solution has been located there is no fear that others can have been missed. The necessary calculations were executed using a Matlab code and, in order to ensure proper convergence, these were checked using an entirely independent Fortran program which relied on a standard nonlinear eigenvalue solver from the NAG suite of routines.

Our first results are concerned with the usefulness of the bounds given by eqn (8). Figure 4 shows the difference between the upper bound $\bar{\psi}(\gamma, K)$ and the actual oxygen concentration at the centre of the slice $u_{0}$. A striking feature of these results is that $\bar{\psi}(\gamma, K)$ is a remarkably good approximation to $u_{0}$ for a wide range of values of both $\gamma$ and $K$. Indeed, for $\gamma<4$ and irrespective of $K$, a range which almost certainly contains the regimes of physiological interest, it appears that the maximum difference between the two values is no more than about $6 \%$ of the oxygen concentration in the surrounding bath. This therefore is a result of much practical interest and shows that although the estimate $\bar{\psi}(\gamma, K)$ was derived from a simple approximation, it represents a useful and powerful result. Meanwhile, Fig. 5 shows the discrepancy between $u_{0}$ and the lower bound of eqn (8a); this is equivalent to the greatest difference between the Michaelis-Menten solution $u(x)$ and the hyperoxia solution $w_{1}(x)$. Clearly, for small $\gamma$, this disparity is quite small over a wide range of values of $K$; as $\gamma$ increases so the constraint on $u_{0}$ provided by this lower bound weakens. Indeed, once $\gamma$ and $\mathrm{K}$ are of appreciable size, the lower bound of eqn (8) is virtually useless for practical purposes. (We note that results in Fig. 5 relate to values $\gamma \leqslant 2$ for with greater $\gamma$ we would have the physically absurd situation of $w_{1}(x)<0$ in the middle of the tissue slice.) The conclusion to be drawn from Figs 4 and 5 is that for the overwhelming range of parameter values the upper bound $\psi(\gamma, K)$ eqn (8) is much more useful than the lower one and, for $O(1)$ values of $\gamma$, the lower bound is only accurate when $K$ is quite small, typically of the order of a few hundredths.

The main motivation for the work described here has been to obtain a description of those circumstances under which the oxygen consumption distribution across a thin tissue slice can be accurately approximated by either the hyperoxia model $w_{1}(x)$ solution (4) or the hypoxia $w_{2}(x)$ solution given by eqn (6). Under even the most careful experimental conditions it is only possible to estimate the parameters $\gamma$ and $K$ of eqn (1) within an error of maybe a few percent. The main reason for this is that these parameters follow from the measurement of oxygen pressure in the tissue and there is often difficulty in assessing the precision of the signal

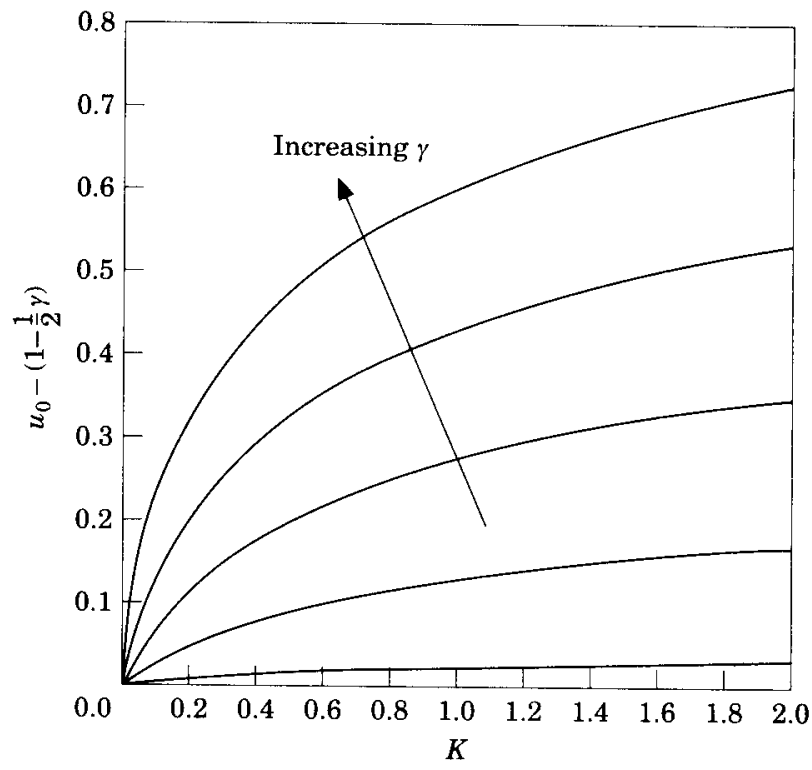

FIG. 5. Graph of the difference between the oxygen concentration at the centre of the slice $u_{0}=u(0 ; \gamma, K)$ and the lower bound $1-\gamma / 2$ as given in (8a). The lines correspond to the parameter values $\gamma=0.1,0.5,1,1.5$ and 2 . 


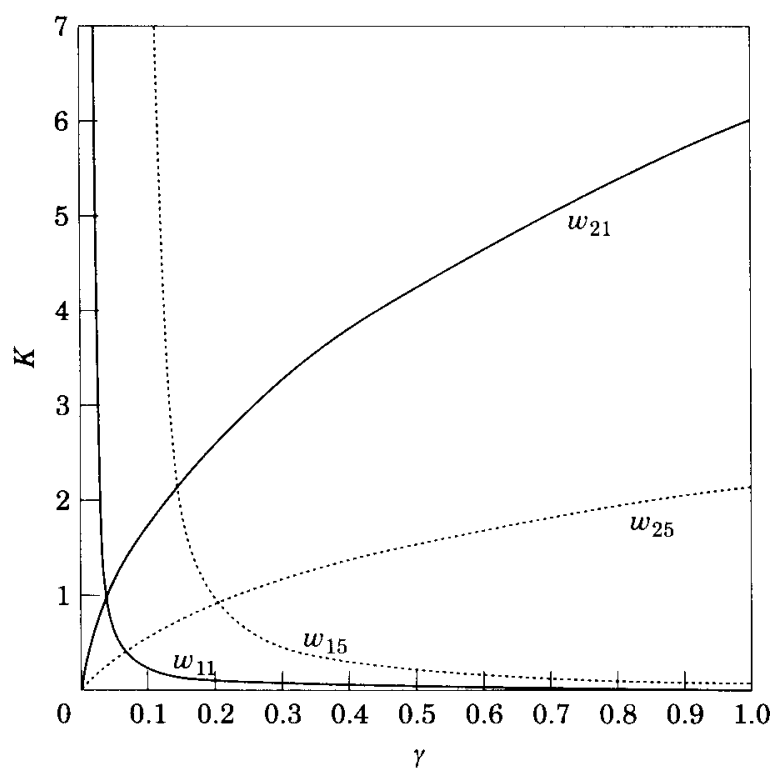

FIG. 6. Plot showing the accuracy of the hyperoxia solution $w_{1}(x)$ and hypoxia solution $w_{2}(x)$ compared with the numerical solution $u(x ; \gamma, K)$ of the Michaelis-Menten equation (1). The regions to the left and below the lines denoted $w_{11}^{\prime}$ and $w_{15}$ are the zones of parameter space in which the difference between $w_{1}(x)$ and $u(x)$ across the slice are respectively less than $1 \%$ and $5 \%$ of the oxygen concentration in the bath. Similarly, the regions to the left and above the lines denoted $w>$ and $x_{25}$ are the domains in which the difference between $w_{2}(x)$ and $u(x)$ across the slice are respectively less than 1 and $5 \%$

strength and the exact position of the microelectrodes in the tissue slice (Lipinski \& Bingmann, 1987; Lipinski, 1992). It is therefore of potential importance to determine the ranges of the control parameters such that $u(x)$ is close to $w_{1}(x)$ or $w_{2}(x)$. In Fig. 6 we provide a sketch of the regions of parameter space in which the difference between $u(x)$ and $w_{1}(x)$ and/or $w_{2}(x)$ is less than 0.01 or 0.05 (that is, this maximum difference is less than $1 \%$ or $5 \%$ of the oxygen concentration outside the tissue slice). In Fig. 6 solid lines mark the divide between parameter regions where the maximum difference between $u(x)$ and $w_{1}(x)$ or $w_{2}(x)$ (as appropriate) is less than or greater than $1 \%$. Dotted lines delimit the corresponding regions at the $5 \%$ accuracy level. The main conclusion to be drawn from the figure is that over a large extent of the parameter space the oxygen uptake characteristics can be well approximated by $w_{1}(x)$ or $w_{2}(x)$. Not surprisingly, as $\gamma$ increases so $w_{1}(x)$ is only useful for a range of small $K$ but $w_{2}(x)$ is helpful for a significant region of sufficiently large $K$.

\section{Conclusions}

In this article we have been interested in the analysis of oxygen consumption across a tissue slice using a Michaelis-Menten model for the necessary diffusion process. Rigorous results concerning the existence and uniqueness of solutions have been established and, importantly, we have proved that the solutions of eqn (1) are necessarily greater than the corresponding hyperoxia and hypoxia forms. These functions, given by eqns (4) and (6) respectively, thereby provide useful lower bounds on our solution. In the case when $\gamma$ is roughly unity, then $w_{1}(x)$ is a useful approximation for $u(x)$ for small $K$ but as $K$ increases so $w_{2}(x)$ becomes increasingly relevant. Additional bounds on the minimum oxygen concentration (which occurs at the centre of the slice) have been deduced and are given by eqn (8).

Most of our numerical solutions and discussion has been deliberately focussed on the physiologically important case when $\gamma=O(1)$. We mentioned in the introduction that for non-physiological experiments then $\gamma$ might be large, sometimes of the order of some hundreds. Then our bounds derived above cease to be particularly useful but in this eventuality other accurate bounds can be easily obtained. Since $0 \leqslant u(x) \leqslant 1$ across the whole of the slice, eqn (1) gives that

$$
\frac{\gamma u}{1+K} \leqslant \frac{\mathrm{d}^{2} u}{\mathrm{~d} x^{2}} \leqslant \frac{\gamma u}{K}
$$

and then, following the arguments of Section 2, it follows that

$$
\frac{\cosh (x \sqrt{\gamma / K})}{\cosh (\sqrt{\gamma / K})} \leqslant u(x ; \gamma, K) \leqslant \frac{\cosh (x \sqrt{\gamma / /(1+K)})}{\cosh (\sqrt{\gamma /(1+K)})}
$$

for all $x \in[-1,1]$. Once $\gamma$ is at all appreciable (e.g. much above about ten) the difference between these two bounding functions is quite tiny and so further work aimed at describing $u(x)$ more precisely is surplus to requirement; at least in the experimental realisable situations for which $K=O(1)$. If $K$ should happen also to be large, calculations along the lines of those summarised in Fig. 6 reveal that the hypoxia solution (6) is in remarkable good agreement with $u(x ; \gamma, K)$ but this case is of little discernible practical relevance.

To sum up, we have deduced a number of useful results concerning the analysis of steady oxygen consumption across a tissue slice. Arguably the most significant result we have established is the potentially important upper bound $\bar{\psi}$ for the minimum oxygen concentration across the slice. Figure 4 illustrates that in a significant majority of parameter space the difference between this theoretical upper bound and the computed Michaelis-Menten solution is no more than a few percent. This minimum concentration value is of great importance in experimental work and 
we have derived a simple yet remarkably accurate estimate for this.

Finally, we mention that an obvious extension of our study would involve the inclusion of unsteadiness and work on this aspect is currently under consideration.

We are indebted to Hans-Gerd Lipinski (Lübeck) who originally suggested the physiological problem to us and guided us through the existing biological literature. Thanks are also due to Dieter Neuffer (Exeter) who supported us by providing some of the first numerical solutions of the equations which led us to reassess our original intuitive notions.

\section{REFERENCES}

Anderson N. \& Arthurs, A. M. (1985). Analytical bounding functions for diffusion problems with Michaelis-Menten kinetics. Bull. Math. Biol. 47, 145-153.

Asaithambi, N. S. \& Garner, J. B. (1989). Pointwise solution bounds for a class of singular diffusion problems in physiology. Appl. Math. Comput. 30, 215-222.

Asaithambi, N. S. \& Garner, J. B. (1992). Taylor series solutions of a class of diffusion problems in physiology. Math. Comput. in Simulations 34, 563-570.

BingmanN, D. \& Kolde, G. (1982). $P O_{2}$-profiles in hippocampal slices of the guinea pig. Exp. Brain Res. 48, 89-96.
Hiltmann, P. \& LoRy, P. (1983). On oxygen diffusion in a spherical cell with Michaelis-Menten uptake kinetics. Bull. Math. Biol. 45 661-664.

KaWashiro, T., NüsSe, W. \& SCheID, P. (1975). Determination of oxygen and carbon dioxide in respiring tissue: results in rat skeletal muscle. Pflügers Arch. 359, 231-251.

LiN, S. H. (1976). Oxygen diffusion in a spherical cell with non-linear oxygen uptake kinetics. $J$. theor. Biol. 60 , 449-457.

LIPINSKI, H. G. (1992). Brain-tissue slice thickness monitored by ion-profile measurement. J. Neuro. Meth. 42, 37.43.

LiPINSKI, H. G. \& BingmanN, D. (1987). Diffusion in slice preparations bathed in unstirred solutions. Brain Res. 437 , 26-34.

MCElwain, D. L. S. (1978). A re-examination of oxygen diffusion in a spherical cell with Michaelis-Menten oxygen uptake kinetics. $J$. theor. Biol. 71, 255-263.

Metzger, H., Hfuber-Metzger, S., Steinacker, A. \& Strüber, J. (1980). Staining $\mathrm{PO}_{2}$ measurement sites in the rat brain cortex and quantitative morphometry of the surrounding capillaries. Pfügers Arch. 388, 21-27.

Michaelis, L. \& Menten, M. I. (1913). Die Kinetik der Invertinwirkung. Biochem. Z. 49, 333-369.

Popel, A. S. (1979). Diffusion in tissue slices with metabolism obeying Michaelis-Menten kinetics. $J$. theor. Biol. $\mathbf{8 0}$, 325-332.

Rashevsky, N. (1960). Mathematical Biophysics, Vol. 1, New York: Dover.

WarburG, O. (1923). Versuche am überlebenden Karzinomgewebe. Biochem. Z. 142, 317-333.

Yамамото, C. (1972). Intracellular study of seizure-like afterdischarges elicited in thin hippocampal sections in vitro. Exp. Neurol. 35, 154-164. 